\title{
La révision de la loi sur l'obligation de diligence du Liechtenstein
}

Fabian Teichmann / Léonard Gerber *

Cet article décrit dans un premier temps l'approche du Liechtenstein quant à la surveillance des prestataires de services liés aux tokens et aux technologies de type blockchain nouvellement régulés, en matière de blanchiment d'argent et de financement du terrorisme. L'entrée en vigueur de la «Blockchain Act» du Liechtenstein le $1^{e r}$ janvier 2020 s'accompagne également d'une révision de la loi sur le devoir de diligence et d'un élargissement du cercle des assujettis aux règles de compliance. Il conclut par une appréciation critique de la révision de la loi sur le devoir de diligence du Liechtenstein.

I. Introduction

II. Approche de la surveillance au Liechtenstein .......................................................39

III. L'élargissement du champ d'application personnel de la SPG ..............................40

1. Les bureaux de change ................................................................................. 41

2. Les courtiers de change .................................................................................. 41

3. Les fournisseurs de tokens ............................................................................... 41

4. Les plates-formes de négociation et les bourses............................................... 41

IV. Appréciation de la révision de la SPG ................................................................42

Citation: $\quad$ Fabian Teichmann/Léonard Gerber, La révision de la loi sur l'obligation de diligence du Liechtenstein, dans: sui-generis 2020, S. 38

URL: $\quad$ sui-generis.ch/120

DOI: $\quad$ https://doi.org/10.21257/sg.120

* Fabian Teichmann (teichmann@teichmann-law.ch), Dr. iur. Dr. rer. pol., LL.M., Rechtsanwalt, Teichmann International (Schweiz) AG; Léonard Gerber (gerber@teichmann-law.ch), Teichmann International (Schweiz) AG. 


\section{Introduction}

1 La représentation digitale de droits sur des objets par le biais de tokens ouvre de nouvelles portes au blanchiment d'argent et au financement du terrorisme notamment en raison de l'anonymat des transactions et des utilisateurs sur les systèmes de transactions basés sur les technologies de type blockchain. ${ }^{1}$ Le Liechtenstein suit de près les problématiques liées aux crypto-monnaies et a mis en place avec la TVTG (Gesetz über Token und VT-Dienstleister) un nouveau cadre légal pour les activités liées aux tokens et aux systèmes de transactions sécurisés. ${ }^{2}$ Cette nouvelle loi, qui est en-

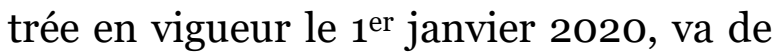
pair avec la révision de la loi sur l'obligation de diligence du Liechtenstein (Sorgfalts-pflichtsgesetz, abrégé SPG). 3 Cet article traite de l'approche de la surveillance, des nouveautés essentielles de la SPG ainsi que de la réception de la révision au Liechtenstein.

\section{Approche de la surveillance au Liechtenstein}

$2 \mathrm{Au}$ niveau international, le Groupe d'action financière (GAFI, abrégé en anglais FATF) attire dans sa recommandation $\mathrm{N}^{\circ} 15$ l'attention des autorités sur les risques de compliance liés aux valeurs virtuelles ainsi qu'aux prestations de serand Potential AML/CFT Risks, 2014, p. 9.

2 Bericht und Antrag der Regierung an den Landtag des Fürstentums Liechtenstein betreffend die Schaffung eines Gesetzes über Token und VTDienstleister und die Abänderung weiterer Gesetze daté du 7 mai 2019 (ci-après Rapport Nr. 54/2019), p. 85s.

3 Cf. Communication du Gouvernement du Liechtenstein du 3 octobre 2019; cf. FAQs zum TVTG, Bericht der FMA; Rapport Nr. 54/2019 (n. 2), p. 305. vices liés aux valeurs virtuelles. 4 Le FATF recommande ainsi de soumettre les prestataires de services liés aux cryptomonnaies à une surveillance, notamment par le biais d'une obligation d'enregistrement ou de licence. 5 Le FATF recommande la surveillance des activités suivantes : L'échange entre les monnaies virtuelles, le transfert, le dépôt, ainsi que l'administration de monnaies virtuelles et la participation à la fourniture de services liés aux monnaies virtuelles. ${ }^{6}$ L’Union Européenne a d'ores et déjà réglementé ce domaine en émettant une $5^{\text {ème }}$ directive anti-blanchiment entrée en vigueur en juillet 2018.7 Cette directive soumet ainsi les prestataires échangeant des monnaies virtuelles contre des monnaies légales et inversement à une obligation de diligence. ${ }^{8}$

3 Dans un souci de mettre en ouvre les standards internationaux de compliance, le gouvernement du Liechtenstein a émis le 7 mai 2019 un rapport explicatif de la TVTG (ci-après Rapport du 7 mai 2019 ou Rapport Nr. 54/2019). Selon celui-ci, la TVTG a pour but de couvrir les risques anticipés par le FATF et l'UE. 9 Au dispo-

4 Rolf Weber/Rainer Baisch, Crowdinvesting mittels Initial Coin Offering - Regulierungsaufgaben im Token-Universum, SZW/RSDA 2019, p. 135154, p. 152.

5 FATF, Guidance for a risk-based approach to virtual assets and virtual asset service providers, p. 55 .

6 FATF, Guidance for a risk-based approach to virtual assets and virtual asset service providers, p. 13 s.

7 Directive (UE) 2018/843 du Parlement européen et du Conseil du 30 mai 2018 modifiant la directive (UE) 2015/849 du 20 mai 2015 relative à la prévention de l'utilisation du système financier aux fins du blanchiment de capitaux ou du financement du terrorisme ainsi que les directives 2009/138/CE du 25 novembre 2009 et 2013/36/UE du 26 juin 2013, ABl. 2018 L 156/43.

8 Rapport Nr. 54/2019 (n. 2), p. 93.

9 Rapport Nr. 54/2019 (n. 2), p. 93. 
sitif de la surveillance du Liechtenstein vient s'ajouter notamment un système d'enregistrement des prestataires liés aux tokens et aux systèmes sécurisés soumis à l'obligation d'enregistrement de la TVTG (art. 12ss TVTG) auprès de l'autorité de surveillance des marchés financiers (Finanzmarktaufsicht, ci-après FMA). ${ }^{10}$

4 Le Liechtenstein met en œuvre les recommandations du FATF depuis longtemps par le biais de sa SPG entrée en vigueur le $1^{\text {er }}$ janvier 1997. ${ }^{11}$ L'obligation de diligence s'impose à toute personne recevant des valeurs patrimoniales issues de tiers à titre professionnel, les tenant en dépôt, ou apportant son aide à leur transfert ou à leur investissement (art. 3 et 4 SPG). ${ }^{12}$ Ainsi, la SPG impose un devoir de contrôle des transactions financières (art. 1 I, 6 I, 7 I, 8 I et 9 I SPG) ainsi qu'un devoir de communication lors de soupçons de transactions liées à un blanchiment d'argent, au crime organisé ou au financement du terrorisme (art. 17 SPG). ${ }^{13}$

Le Rapport du 7 mai 2019 estime que la SPG actuelle mettant en œuvre les standards internationaux constitue déjà un outil approprié pour lutter efficacement contre le blanchiment d'argent en lien avec les systèmes sécurisés. ${ }^{14}$ Le gouvernement du Liechtenstein compte donc suivre les recommandations du FATF en élargissant le champ d'application personnel de la SPG en soumettant les pres-

10 Rapport Nr. 54/2019 (n. 2), p. 97.

11 Norbert Seeger/Lars Heidbrink/Marco Tanner, Deutsches und europäisches Bank- und Kapitalmarktrecht, p. 2447-248o, p. 2462.

12 Seeger/Heidbrink/Tanner (n. 11), p. 2463.

13 Seeger/Heidbrink/Tanner (n. 11), p. 2462.

14 Rapport Nr. 54/2019 (n. 2), p. 96. tataires de services en lien avec des monnaies virtuelles à une obligation de diligence (art. 2 et 3 I SPG). ${ }^{15}$ À cet égard, la FMA sera chargée de la surveillance des prestataires de systèmes sécurisés en lien avec la SPG (art. 23 I let. a SPG). ${ }^{16}$

\section{L'élargissement du champ d'application personnel de la SPG}

6 La révision du SPG concerne cinq nouveaux types d'acteurs. ${ }^{17}$ D'une part, les négociants de biens, effectuant des transactions en cash liées aux cryptomonnaies, à partir d'un montant de $10^{\prime}$ Ooo CHF (art. 3 I let. q SPG).18 D'autre part, la SPG s'applique à une partie des prestataires professionnels soumis à enregistrement en vertu de la TVTG (art. 3 I let. r SPG), à savoir les fournisseurs de tokens de paiement, les dépositaires de tokens, les dépositaires de clés privées, le validateur physique, le protecteur de systèmes sécurisés et le courtier de systèmes sécurisés. ${ }^{19}$ Une troisième catégorie concerne, sous certaines conditions, les fournisseurs de tokens de paiement non-soumis à la TVTG (art. 3 I let. s SPG). Les deux dernières catégories comprennent les gérants de plates-formes de monnaies virtuelles respectivement de tokens de

15 Rapport Nr. 54/2019 (n. 2), p. 305.

16 Rapport Nr. 54/2019 (n. 2), p. 309; voir également Seeger/Heidbrink/Tanner (n. 11), p. 2468.

17 Notons que la présence physique au Liechtenstein constitue le critère de rattachement principal pour l'application de la TVTG (art. 11 TVTG) et donc indirectement de la SPG. Les entreprises dont le siège ou le domicile est à l'étranger ne doivent pas être soumises à la TVTG, une exception est toutefois prévue lorsque l'entreprise étrangère établi un automate de change au Liechtenstein (art. $16 \mathrm{SPG}$ ); voir à cet égard Rapport Nr. 54/2019 (n. 2), p. 85s.

18 Rapport Nr. 54/2019 (n. 2), p. 368s.

19 Rapport Nr. 54/2019 (n. 2), p. 305. 
paiement (art. 3 I let. t SPG) ainsi que les bourses monétaires électroniques (art. 3 I let. u SPG). ${ }^{20}$ Le Rapport du 7 mai 2019 se concentre principalement sur les acteurs suivants que nous proposons d'étudier de plus près.

\section{Les bureaux de change}

7 Depuis 2017, la SPG s'applique aux bureaux de change lorsqu'ils échangent des monnaies virtuelles contre des monnaies légales à partir d'une somme de 1'Ooo CHF (Wechselstube, au sens des art. 3 I let. f et art. 2 I let. i SPG). ${ }^{21}$ Les bureaux de change constituent au sens de la SPG des vaisseaux pour l'échange de monnaies légales, en excluant toutefois les échanges entre monnaies virtuelles (art. 2 I let. i SPG). ${ }^{22}$

\section{Les courtiers de change}

8 En réponse à la lacune citée ci-dessus, la TVTG introduit la prestation de courtier de change de système sécurisé (VTWechseldienstleister au sens de l'art. 2 let. r TVTG, ainsi que l'art. 2 I let. ibis SPG) impliquant le négoce de monnaies virtuelles contre des moyens de paiement légaux et inversement ainsi qu'entre monnaies virtuelles comme les tokens. ${ }^{23}$ Ceux-ci sont soumis à l'obligation de diligence par le biais de l'art. 3 I let. r SPG peu importe le montant des transactions. ${ }^{24}$ Ces courtages peuvent également survenir par le biais d'automates physiques de change dont le gérant sera soumis à l'obligation de diligence à partir

\footnotetext{
Rapport Nr. 54/2019 (n. 2), p. 305.

Rapport Nr. 54/2019 (n. 2), p. 93.

Rapport Nr. 54/2019 (n. 2), p. 302.

Rapport Nr. 54/2019 (n. 2), p. 82.

Rapport Nr. 54/2019 (n. 2), p. 97s.
}

d'une somme de 1'ooo CHF (art. 5 II let. h SPG). 25

\section{Les fournisseurs de tokens}

9 S'agissant des fournisseurs de tokens de paiement (Token-Emittent au sens de l'art. 2 I let. I TVTG) ${ }^{26}$, ceux soumis à l'obligation d'enregistrement selon l'art. 12 TVTG sont également soumis à l'obligation de diligence en vertu de l'art. 3 I let. r SPG peu importe la somme des transactions effectuées. ${ }^{27}$ En revanche, les fournisseurs de tokens nonsoumis à l'obligation d'enregistrement en vertu de l'art. 12 TVTG ${ }^{28}$ sont soumis à l'obligation de diligence lorsqu'ils exécutent des transactions à hauteur de 1'Ooo CHF ou plus (art. 3 I let. s SPG). ${ }^{29}$ Enfin, s'agissant des fournisseurs émettant d'autres types de tokens représentatifs de biens et non de tokens de paiement, ceux-ci ne sont pas soumis à l'enregistrement mais seront soumis à la SPG en vertu de l'art. 3 I let. q SPG en tant que négociants de biens. ${ }^{30}$

\section{Les plates-formes de négociation et les bourses}

10 Les plates-formes de négociation organisée (Organised Trading Facility, OTF) ${ }^{31}$, les systèmes multilatéraux de négociation

25 Rapport Nr. 54/2019 (n. 2), p. 308.

26 À ne pas confondre avec le créateur de tokens (Token-Erzeuger au sens de l'art. 2 I let. $\mathrm{m}$ TVTG) qui n'offre pas publiquement les tokens et n'est donc pas soumis aux obligations relatives à la feuille d'information de base (art. 30ss. TVTG).

27 Rapport Nr. 54/2019 (n. 2), p. 105s.

28 En cas d'exercice de l'activité à titre privé ou en nom propre ou en cas d'émission de tokens d'une valeur inférieure à 5 mio. de CHF en vertu de l'art. 12 TVTG.

29 Rapport Nr. 54/2019 (n. 2), p. 105s.

30 Rapport Nr. 54/2019 (n. 2), p. 102.

31 Voir à cet égard Rolf Sethe, Kommentar zum [schweizerischen] Finanzmarktinfrastrukturgesetz, Zürich 2017, Finfrag 26 N 35. 
(Multilateral Trading Facility, MTF) ${ }^{2}$ ainsi que les bourses (Börsen)33 nécessitent une licence de banque ou de maisons de titres. Lorsqu'ils négocient des tokens représentant digitalement des titres, ils seront également soumis à la SPG en tant qu'intermédiaires financiers (art. 3 I let. a et $\mathrm{b}$ SPG).34 Il en va de même pour les plates-formes de négociation de tokens représentant des droits sur des objets physiques s'ils sont structurés en tant qu'instruments financiers.

11 À défaut d'être soumis à une autorisation de banque ou de maisons de titres, les gérants de plates-formes de négociation de monnaies virtuelles (Betreiber von Handelsplattformen für virtuelle Währungen bzw. Zahlungstoken au sens de l'art. 2 I let. zquater SPG) seront toutefois soumis à l'obligation de diligence en vertu de l'art. 3 I let. t SPG.35 De plus, les gérants de bourses monétaires électroniques (Anbieter von elektronischen Geldbörsen au sens de l'art. 2 I let. $z^{\text {ter }}$ SPG) présentent un risque accru de blanchiment d'argent car ils garantissent l'anonymat des utilisateurs tout en ayant accès aux clés privées des utilisateurs et sont donc expressément soumis à la SPG (art. 3 I let. u SPG). ${ }^{6}$ Lorsque les monnaies virtuelles ne représentent pas des instruments financiers mais des objets physiques, une soumission à la SPG est possible en qualité de dépositaire de tokens ou de dépositaire de clés-privées (art. 3 I let. $r$ SPG). 37

Voir à cet égard Sethe (n. 31), Finfrag 42 N 2.

3 Voir à cet égard Sethe (n. 31), Finfrag $26 \mathrm{~N} 21$.

Rapport Nr. 54/2019 (n. 2), p. 76s et 105.

Rapport Nr. 54/2019 (n. 2), p. 76s et 105.

Rapport Nr. 54/2019 (n. 2), p. 369.

Rapport Nr. 54/2019 (n. 2), p. 104s.

\section{Appréciation de la révision de la SPG}

12 L'association des banquiers du Liechtenstein salue de son côté fondamentalement l'initiative du gouvernement de soumettre les prestataires de services liés aux systèmes sécurisés à la loi sur le devoir de diligence. $3^{8}$ Toutefois, il faut relever en partie le manque de règles adaptées aux technologies DLT et blockchain s'agissant des know your customer rules 39 , des processus de surveillance ainsi que de règles sur la surveillance. 40 Les normes de la SPG sont peu compatibles avec la structure exclusivement digitale des systèmes basés sur la blockchain et les registres décentralisés. ${ }^{41} \mathrm{En}$ effet, la SPG révisée prévoit uniquement à cet égard que «l'historique des transactions en lien avec des monnaies virtuelles, de leur acquisition et de la contre-prestation lors des transactions devra être contrôlé par un système informatique».42 Par conséquent, avec l'association des banquiers du Liechtenstein, on aurait souhaité que la SPG fût complétée sur la base d'une étude plus approfondie des risques pour la sécurité informatique. Le gouvernement donne en effet raison à l'association et prévoit de compléter la SPG notamment à l'aide de guides et de directives. 43

13 Quant aux inquiétudes exprimées par l'association à propos des ressources nécessaires pour faire face à la soumission

$38 \overline{\text { Voir à ce sujet le rapport Nr. 54/2019 (n. 2) }}$, p. 306.

39 À ce sujet voir Seeger/Heidbrink/Tanner (n. 11), p. 2464.

40 Rapport Nr. 54/2019 (n. 2), p. 307.

41 Rapport Nr. 54/2019 (n. 2), p. 307.

42 Rapport Nr. 54/2019 (n. 2), p. 308, voir à cet égard le nouvel art. 9b SPG.

43 Rapport Nr. 54/2019 (n. 2), p. 307. 
de cinq nouveaux types de prestataires à la $\mathrm{SPG}, 44$ on rappellera que le gouvernement du Liechtenstein dans son Rapport du 7 mai 2019 renvoie aux conditions personnelles, financières et organisationnelles des prestataires soumis à la TVTG notamment s'agissant de leur capital minimum (art. 16ss. TVTG). 45 Selon le Rapport du 7 mai 2019, ces conditions d'enregistrement permettraient de renforcer la réputation de la place financière liechtensteinoise et garantir la protection des créanciers sans toutefois freiner l'innovation et mettre des obstacles trop importants aux entreprises actives dans les crypto-monnaies. 46

Nous saluons également la renonciation à une surveillance prudentielle de la solvabilité des prestataires qui conduira à des coûts de surveillance moins onéreux à charge des prestataires directement surveillés. Ils vont de $250 \mathrm{CHF}$ à 1'ooo $\mathrm{CHF}$ par an selon le type de prestataire avantageant ainsi les prestataires soumis à la TVTG par rapport aux autres intermédiaires assujetti à la surveillance de la FMA.47 À cet égard, une requête de la FMA quant à une augmentation à 10'0oo CHF par an des frais annuels requis pour la surveillance fut rejetée par le gouvernement liechtensteinois. 48 À notre sens, la position du gouvernement se justifie au regard de la nature des entreprises actives dans la technologie blockchain et les crypto-monnaies, celles-ci constituant le plus souvent des start-ups ne possédant pas les mêmes moyens financiers que les banques ou les maisons de titres.

44 Rapport Nr. 54/2019 (n. 2), p. 306.

45 Rapport Nr. 54/2019 (n. 2), p. 307.

46 Rapport Nr. 54/2019 (n. 2), p. 306.

47 Rapport Nr. 54/2019 (n. 2), p. 312.

48 Rapport Nr. 54/2019 (n. 2), p. 312.
15 Notons toutefois que les mécanismes de compliance sont largement contournables.49 Malgré les efforts internationaux déployés pour les éradiquer, tant le blanchiment d'argent que le financement du terrorisme demeurent des problèmes globaux.50 Des mécanismes de compliance basés sur la responsabilité des intermédiaires financiers sont inadéquats car il ne s'agit pas d'organes d'exécution de la loi et ils ne sont ainsi pas investis des moyens nécessaires pour déterminer l'arrière-plan économique des transactions de leurs clients. ${ }^{11}$ Bien que les acteurs privés comme les banques ou les prestataires de services liés aux cryptomonnaies peuvent apporter leur soutien à la lutte contre le blanchiment d'argent, le financement du terrorisme et le crime organisé, il est excessif de leur déléguer la responsabilité principale dans l'accomplissement de cette tâche. Une solution serait de les doter de moyens permettant d'analyser en profondeur l'arrière-plan des transactions de leurs clients comme la surveillance électronique ou la possibilité d'effectuer des contrôles sur place. Ainsi, l'élargissement de la SPG du Liechtenstein basée sur la responsabilité des intermédiaires financiers pour lutter contre ces crimes, toutefois sans les doter des moyens néces-

$49 \overline{\text { Voir à cet égard Fabian Teichmann, Umge- }}$ hungsmöglichkeiten der Geldwäschereipräventionsmassnahmen, Schulthess, Zürich 2016, p. 2s.

5o Jackie Harvey, «Compliance and reporting issues arising for financial institutions from money laundering regulations: A preliminary cost benefit study, 2004, Journal of Money Laundering Control, p. 339; Petrus Van Duyne, Money Laundering: Estimates in Fog, 1994, Journal of Financial Crime, vol. 2 No. 1, p. 62; John Walker, How big is global money laundering ?, 1999, Journal of Money Launderiung, p. 36.

${ }^{51}$ Fabian Teichmann, Money-Laundering and Terrorism-Financing Compliance - Unsolved Issues, 2018, Journal of Money Laundering Control, p. 5. 
saires pour y parvenir, ne fera que perpétuer les faiblesses actuelles des mesures de compliances. 